\title{
Comparison of dynamic models for non-contact micromanipulation based on dielectrophoretic actuation
}

\author{
Vladimir Gauthier, Aude Bolopion and Michaël Gauthier
}

\begin{abstract}
Several approaches are proposed in the literature to calculate the drag force, the electric field and the induced dielectrophoretic force. This paper analyzes the performances of various models for closed loop control of dielectrophoretic systems in comparison with experiments. This article compares their performance in terms of accuracy, computation time, and memory consumption. Four classical approaches are available to calculate the electric field. Their performances are analyzed in the paper. We have shown that combining the dipolar model of dielectrophoresis force with an anisotropic drag force (integrating the wall-effect) provides an interesting ratio precision/computation time. This paper provides an original comparison of several models described in literature whose performances have been compared with experiments.
\end{abstract}

\section{INTRODUCTION}

Manipulation of micro-objects in fluid is of great interest, especially in biological applications. In this framework, a great variety of systems has been developed [1] which includes the dielectrophoresis (DEP) systems. The DEP physical principle enables to manipulate a dielectric microobject placed in an AC electric field [2]. Under certain conditions, dielectric objects (including biological cells) are polarized and are attracted toward local extrema of the electric field. The electric field are commonly produced by electrodes sputtered on a substrate. Most of the systems are dedicated to statistical sorting of cells, but the new generation is focused on single cell positioning [3].

To guarantee that a single micro-object is positioned accurately, closed-loop control must be implemented. Along the trajectory, the micro-object positions can be measured with cameras [4], event based sensors [5] or by impedance spectrometry [6]. The control law requires a model of the system characterized by high non-linearities. This model is based on the forces applied on the particle (dielectrophoretic force, drag force, etc.). The first step of the model consists in the calculation of the electric field around the manipulated object. Different approaches have been proposed such as the electric field superposition [7], the charge distribution superposition [8], the Green's function [9] or the Fourier series

Authors are with FEMTO-ST Institute, AS2M department, Univ Bourgogne Franche-Comté, CNRS, 24 rue Alain Savary, 25000 Besancon, France, vladimir.gauthier@femto-st.fr

This work has been supported by the EUR EIPHI program (Contract No ANR-17-EURE-0002), by the French Agence Nationale de la Recherche and the Swiss National Science Foundation through the CoDiCell project (contract "ANR-17-CE33-0009-01"), by the MiMedi project funded by BPI France (grant No. DOS0060162/00) and the European Union through the European Regional Development Fund of the Region Bourgogne-FrancheComte (grant No. FC0013440), by the French RENATECH network and its FEMTO-ST technological facility, by the Collegium SMYLE (SMart SYstems for a better LifE) and by the Region Bourgogne-Franche-Comte.

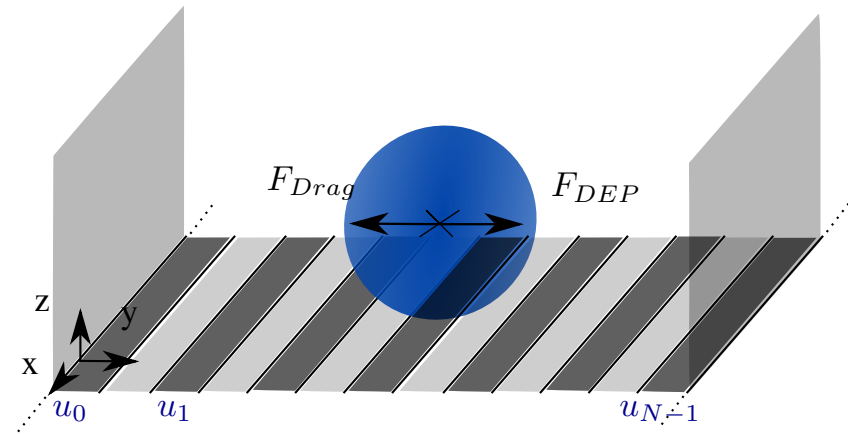

Fig. 1: Benchmark system used for the comparison: an array of parallel electrodes in a fluidic channel to control the lateral position of microparticles by applying a dielectrophoretic force. The electrode's width, $l$, is $5 \mu \mathrm{m}$ or $10 \mu \mathrm{m}$ and the particles have a radius, $a$, between $5 \mu \mathrm{m}$ and $10 \mu \mathrm{m}$.

[10]. The calculation of the dielectrophoretic force depends on assumptions on the electric field's non-uniformity, leading to multipolar model. The other predominant force is the drag force induced by the liquid, and several expressions are also proposed in the litterature [11], [12].

To use these models to perform closed-loop control they must meet several requirements in terms of accuracy, computation time and memory requirement. This article proposes to analyze and compare these models. Several experimental particle trajectories are taken as references. This article will thus provide a comprehensive analysis for people interested in implementing closed-loop control based on dielectrophoretic actuation.

This paper is organized as follows. Section II provides the quasi-static model of the system. Section III compares several models of drag and dielectrophoretic forces with experiments. Section IV analyses different approaches to compute the electric field. Section V concludes the paper.

\section{Motion OF A PARTICLE IN AN AC ELECTRIC FIELD}

To compare different models of the drag and dielectrophoretic forces, as well as different approaches to compute the electric field, experimental data will be taken as references. They will be obtained from a benchmark described in Figure 1. It is composed of an array of parallel electrodes in a fluidic channel. This configuration is of interest for example to sort micro-objects, or biological cells. The microobjects flow through the channel along the $x$ direction due to the fluid motion. The lateral position $y$ of the micro-object is controlled by the electric potential on each electrode. 
TABLE I: Order of magnitude of the velocity induced by each force acting on the benchmark experiments. Those values ares comptuted for ethanol and water. For other liquids, refer to formula given in [13].

\begin{tabular}{|c|c|}
\hline Force & Velocity \\
\hline \hline Gravity - Buoyancy & $10^{-4} \mathrm{~m} \mathrm{~s}^{-1}$ \\
\hline Dielectrophoresis & $10^{-3} \mathrm{~m} \mathrm{~s}^{-1}$ \\
\hline Brownian motion & $10^{-7} \mathrm{~m} \mathrm{~s}^{-1}$ \\
\hline AC electro-osmosis & $10^{-5} \mathrm{~m} \mathrm{~s}^{-1}$ \\
\hline Electrothermal flow & $10^{-9} \mathrm{~m} \mathrm{~s}^{-1}$ \\
\hline
\end{tabular}

Deviating the position of the objects enables to collect them in different reservoirs to sort them. This paper focuses on modeling the lateral displacement induced by the electrode array. In the benchmark experiments, the electrode's width, $l$, is $5 \mu \mathrm{m}$ or $10 \mu \mathrm{m}$ and the spherical micro-objects (particles) have a radius, $a$, between $5 \mu \mathrm{m}$ and $10 \mu \mathrm{m}$.

\section{A. Dynamic model}

In the following, we consider that the liquid in the microchannel is static, and that the trajectory of the particule is consequently in the plane defined by $(\vec{y}, \vec{z})$. In the general case, the forces applied on the particle are the fluid drag force, gravity-buoyancy, dielectrophoresis force and Brownian motion. Moreover, the medium can be subjected to $\mathrm{AC}$ electro-osmosis and electrothermal flow. Based on the work of Castellanos et al. [13], it is possible to compute the relative importance of these forces on the velocity of the particle. Table I summarizes the order of magnitude of the particule velocity induced by each force.

AC electro-osmosis, Brownian motion and electrothermal flow are at least two orders of magnitude less than the dielectrophoretic force, and one order of magnitude less than the joint effect of gravity and buoyancy. According to this calculation, these three effects will be neglected in our case.

For a particle subjected to gravity-buoyancy $\vec{F}_{g}$, dielectrophoresis $\vec{F}_{D E P}$ and fluid drag $\vec{F}_{d r a g}$, Kharboutly et al. demonstrated that the inertial term can be neglected in Newton's second law [8]. Thus, the motion of the particle can be deduced from:

$$
0=\vec{F}_{g}+\vec{F}_{d r a g}+\vec{F}_{D E P} .
$$

The gravity-buoyancy force is given by:

$$
\vec{F}_{g}=\frac{4}{3} \pi a^{3}\left(\rho_{m}-\rho_{p}\right) g \vec{z},
$$

where $a$ is the particle's radius, $\rho_{m}$ (resp $\rho_{p}$ ) is the fluid density (resp. particle density) and $-g \vec{z}$ the gravitational acceleration. The next sections present various expressions of the drag and dielectrophoretic forces proposed in the literature.

\section{B. Model of the drag force}

The classical model of the drag force applied on a spherical object is given by the Stockes' law. An extended version of this law has been proposed in [11], [12]:

$$
\vec{F}_{\text {drag }}=-6 \pi \mu a\left(\lambda_{y} \dot{y} \vec{y}+\lambda_{z} \dot{z} \vec{z}\right),
$$

where $\mu$ is the dynamic viscosity and $\lambda_{y}$ and $\lambda_{z}$ are correction factors enabling to model the impact of the substrate close to the particle (wall effect). These correction factors are defined in [11, Eq. (1)] and [12, Eq. (3.2)]:

$$
\begin{aligned}
\lambda_{y}= & {\left[1-\frac{9}{16} \frac{a}{z}+\frac{1}{8}\left(\frac{a}{z}\right)^{3}-\frac{45}{256}\left(\frac{a}{z}\right)^{4}-\frac{1}{16}\left(\frac{a}{z}\right)^{5}\right]^{-1}, } \\
\lambda_{z}= & \frac{4}{3} \sinh \alpha \sum_{n=1}^{\infty} \frac{n(n+1)}{(2 n-1)(2 n+3)} \\
& {\left[\frac{2 \sinh (2 n+1) \alpha+(2 n+1) \sinh 2 \alpha}{4 \sinh ^{2}\left(n+\frac{1}{2}\right) \alpha-(2 n+1)^{2} \sinh ^{2} \alpha}-1\right] }
\end{aligned}
$$

where

$$
\alpha=\cosh ^{-1}(1+(z-a) / a),
$$

$z$ is the altitude of the center of gravity of the particle above the substrate. As the drag coefficients are different along $y$ and $z$, this general model is called "anistropic drag force" in the following of this paper. The isotropic classical Stockes' law represents the particular case of Eq 3 considering the assumption $\lambda_{y}=\lambda_{z}=1$. This simplified model is called "isotropic drag force" in the following. Section III-B discusses the influence of the anistropy of the drag force on particle trajectories.

\section{Model of the dielectrophoretic force}

Close to the electrodes, the electric field is highly nonuniform. The force induced by a highly non-uniform electric field on a uniform spherical dielectric particle can be derived using Maxwell stress tensor [14] or effective multipole moment [15]. Maxwell stress tensor approach is regarded as the most rigorous. However, Michálek [7] shows that for a spherical particle over parallel electrode array the quadripolar approximation shows results similar to Maxwell stress tensors. Maxwell stress tensors being too long to compute for closed-loop control, effective multipole moment are usually used. The quadripolar approximation of the $i$ component, $i \in\{y, z\}$, of the dielectrophoretic force induced by an electric field $\vec{E}$ can be found in [16, Eq. (8)] as:

$$
\begin{aligned}
& F_{D E P, i}= \\
& 4 \pi \varepsilon_{m} a^{3}\left[K^{(1)} E_{m} \frac{\partial E_{i}}{\partial x_{m}}+\frac{K^{(2)} a^{2}}{6} \frac{\partial E_{m}}{\partial x_{n}} \frac{\partial^{2} E_{i}}{\partial x_{n} \partial x_{m}}\right] .
\end{aligned}
$$

Equation (6) uses the Einstein summation convention: all repeated indexes are summed. The real part of the generalized Clausius-Mossotti factor $K^{(n)}$ is defined as:

$$
K^{(n)}=\operatorname{Re}\left(\frac{n\left(\varepsilon_{p}^{*}-\varepsilon_{m}^{*}\right)}{n \varepsilon_{p}^{*}+(n+1) \varepsilon_{m}^{*}}\right)
$$


TABLE II: Experiments performed to obtain reference data

\begin{tabular}{|l|c|c|c|}
\hline & Electrodes width $l$ & Part. radius $a$ & Part. material \\
\hline Experiment 0 & $10 \mu \mathrm{m}$ & $5.1 \mu \mathrm{m}$ & Borosilicate \\
\hline Experiment 1 & $5 \mu \mathrm{m}$ & $5.1 \mu \mathrm{m}$ & Borosilicate \\
\hline Experiment 2 & $5 \mu \mathrm{m}$ & $8.7 \mu \mathrm{m}$ & Borosilicate \\
\hline Experiment 3 & $5 \mu \mathrm{m}$ & $10.2 \mu \mathrm{m}$ & Polystyrene \\
\hline
\end{tabular}

where $\varepsilon_{m}^{*}$ and $\varepsilon_{p}^{*}$ are the complex permittivities of the medium and the particle, respectively. These are defined as $\varepsilon^{*}=\varepsilon+\sigma j \omega$ with $\varepsilon$ the permittivity, $\sigma$ the conductivity and $\omega=2 \pi f$ the angular frequency of the harmonic electric field. Equation (6) describes the quadripolar expression of the DEP force. The dipolar expression of the DEP force is obtained form Eq (6) by neglecting the terms $\frac{\partial^{2} E_{i}}{\partial x_{n} \partial x_{m}}$. The comparison between both expressions are defined in sec. IIIC.

The next section discusses the model of the drag force, and in particular the drag force anisotropy (Eq. 4 and Eq. 5 ) as well as the model of the dielectrophoretic force (Eq. 6), difference between quadripolar and dipolar model, compared to experimental data. Section IV focuses on different approaches to compute the electric field.

\section{COMPARISON BETWEEN MODELS AND EXPERIMENTS}

Using equations (1) to (6) the trajectory of the particle can be defined considering its initial position and the electric field in the workspace. The objective of this section is to compare the computed trajectories of the models presented in the previous section with experiments. This comparison requires to compute the electric field in the workspace. Different methods to compute this electric field can be used providing a very similar computed electric field but requiring different calculation times and memory requirements. The comparison of the different ways to compute the electric field will be presented in section IV. In this section, the electric field in the workspace is calculated using the "Fourier Series method" [10].

\section{A. Experimental data}

The models discussed in this paper are compared with experimental data, considered as the reference. Four experiments listed in Table II are reported in this paper. The four experiments enable to test trajectories for various parameters such as different electrode width $l$, particle radius $a$ and particle materials. The experiment are conducted in a petri dish with an open channel. To avoid bubble formation, ethanol is used. For each experiment the motion is recorded at 1000 frames per second with a pixel resolution of $1.44 \mu \mathrm{m}$ allowing accurate off-line tracking. At $t_{0}$, a step voltage of $5 \mathrm{~V}_{\text {rms }}$ at $500 \mathrm{kHz}$ is set on one electrode close to the object, the voltage of others electrodes are set to zero.

\section{B. Impact of the drag force anisotropy}

Fig 2 describes the value of the factors $\lambda_{y}$ and $\lambda_{z}$ in function of the ratio $d / a$ between $d=z-a$ the particlesubstrate distance and $a$ the particle radius. As expected,

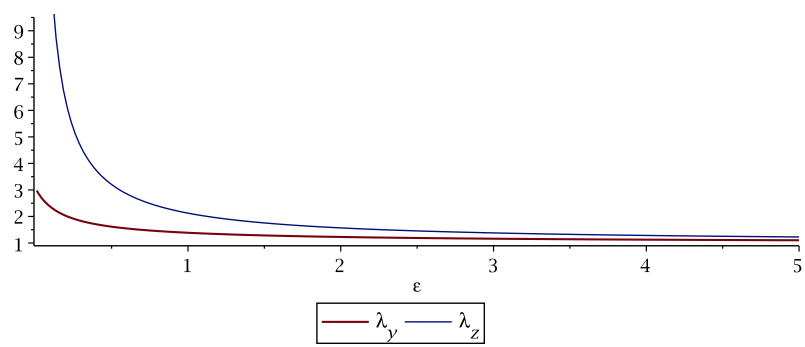

Fig. 2: Value of the Stockes' law correction for wall effect depending on the ratio $\epsilon=d / a$ between $d=z-a$ the particle-substrate distance and $a$ the particle radius.

when the particle goes close to the substrate, the correction factors increase and the drag force increases too $\mathrm{Eq}(3)$.

As $\lambda_{z}$ is always greater than $\lambda_{y}$ (e.g. $\lambda_{z} / \lambda_{y}=1.53$ for $d / a=1$ ), the drag coefficient along $y$ is smaller than the one along $z$. This anisotropy induced by the substrate (walleffect) has a strong impact on the particle trajectory. Indeed, the same force amplitude along $y$ and $z$ induce a velocity along $z$ smaller than the velocity along $y$. In other words, the wall-effect tends to favor the movements parallel to the plane. Figure 3 illustrates this effect. It shows the position of the particle (experiment 0 ) as a function of time. Figure 3(b) represents the altitude of the particle predicted by models. The models taking into account the drag force anisotropy predicts a trajectory with a lower height than the models based on isotropic drag force. Moreover, the height of the particle has a significant impact on the magnitude of the DEP force: the lower is the particle, the higher the electric field gradients are, the higher the DEP force is. Consequently, the anisotropic drag force model predicts a trajectory along $y$ with a higher velocity than the isotropic model.

The anisotropic model shows a good coherence with the experimental measurement. All further simulations uses the anisotropic drag force model.

\section{Multipolar dielectrophoresis}

While computing the dielectrophoretic force, higher order terms are commonly neglected based on the dipole approximation. However, in theory, this dipole approximation is valid only if the spatial variations of the electric field are small compared to the particle size [15]. In this section the influence of higher order multipoles (quadrupoles) is evaluated with respect to experimental data.

Figure 4 represents the position of the particle obtained both experimentally and using the proposed models with the dipolar and quadripolar approximations. Experimental conditions are the following. The electrode size is decreased to $5 \mu \mathrm{m}$ and spherical particles with radius up to $10.2 \mu \mathrm{m}$ are tested according to Table II. A step voltage of $5 \mathrm{~V}$ is applied on an electrode at $t_{0}=10 \mu \mathrm{s}$. To compute the trajectories obtained from the model (Eq. 1) the anisotropic drag force is considered, as discussed in the previous section. To compute the dielectrophoretic force the electric field is obtained from the Fourier series method [10]. This choice is discussed in 


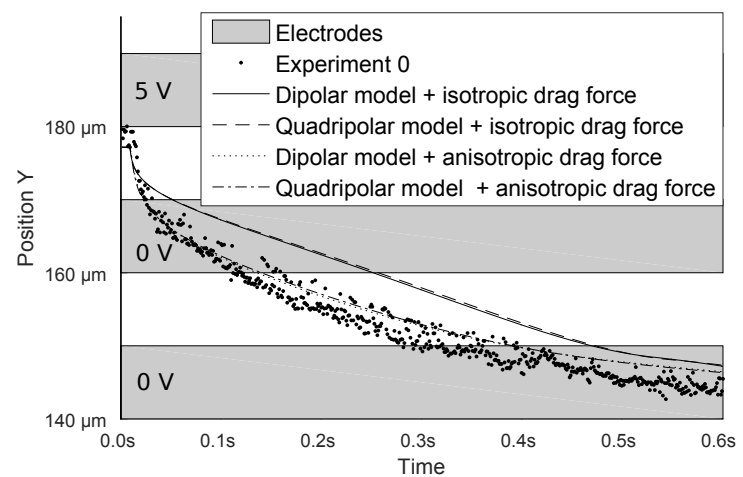

(a) Lateral position along $\vec{y}$ axis

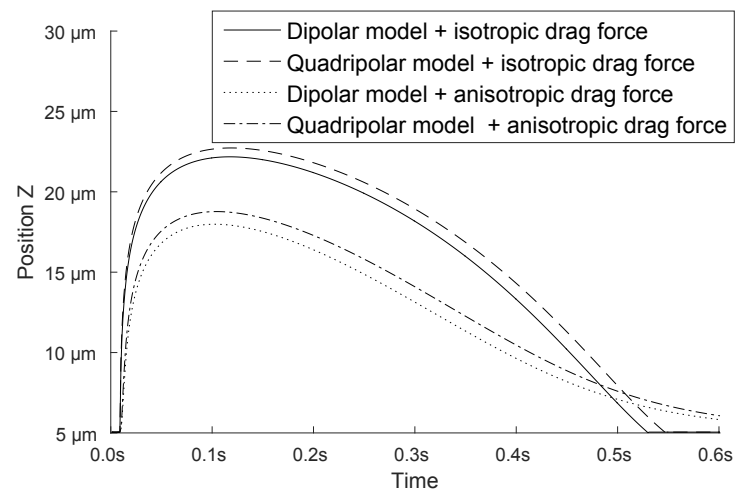

(b) Altitude along $\vec{z}$ axis

Fig. 3: Experiment 0: Position of the particle in response to a step voltage. Models computing dipolar and quadripolar expressions of the DEP force and isotropic and anisotropic drag forces are compared to experiments. As Eq (5) leads to infinite drag force at the initial position, particle-substrate distance $d=0$, during the first five microseconds of simulation the following expression of $\alpha$ is considered in Eq (5): $\alpha_{d=0}=\cosh ^{-1}(1+(z-0.99 a) / a)$.

Sec. IV. The trajectories of the particle obtained for both the dipolar and quadripolar approximations are plotted in Fig. 4 and compared with experiments.

According to Figure 4, even if the electric field is highly non-uniform, the trajectories obtained from the models and the experiments are very close. As expected, the quadripolar model is slightly more accurate when predicting the particle trajectory. However, it takes twice as long to compute. Since computation time is a critical issue for closed loop control the dipolar approximation is retained in the following.

\section{ELECTRIC FIELD COMPUTATION}

The previous section analyzes the influence of different models of the dielectrophoretic force. However, it was assumed that the electric field was known. This section discusses different approaches to compute the electric field in terms of computing resources and accuracy.

There are three different approaches to compute the electric field: numerical models, semi-analytical models and analytical models. Numerical models, such as finite element

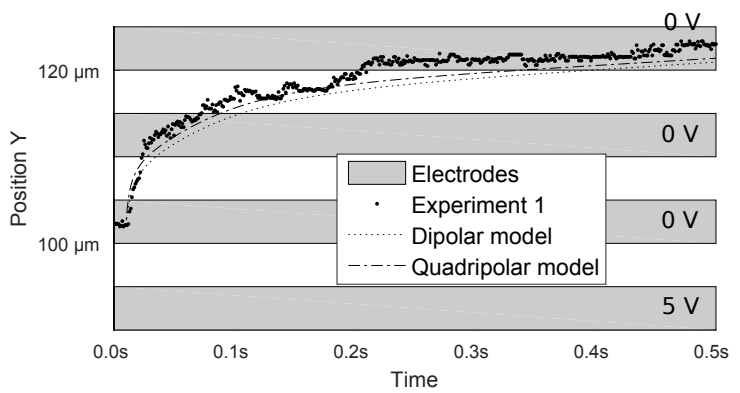

(a) Experiment 1 - lateral position along $\vec{y}$ axis

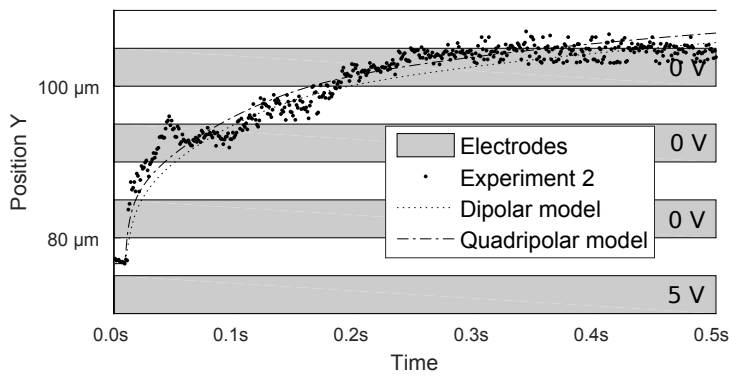

(b) Experiment 2 - lateral position along $\vec{y}$ axis

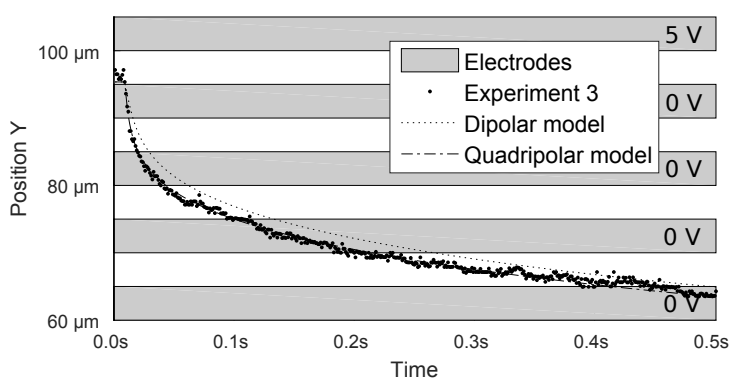

(c) Experiment 3 - lateral position along $\vec{y}$ axis

Fig. 4: Position of the particle in the channel with respect to time in response to a step voltage. The dipolar and quadripolar approximations are compared to experiments for particles having different radius.

methods, require too much computation time to be usable for closed loop control of a particle motion using dielectrophoresis. They are not considered in this paper.

\section{A. Semi-analytical methods}

Semi-analytical models use the superposition principle to extrapolate the electric field from a base of pre-computed numerical solution [8], [7].

1) Superposition principle on the electric field: The superposition principle can be applied directly to the electric field and its derivative issued from each electrode, leading to:

$$
E(x, y, z)=\sum_{n=1}^{N} E_{n}(x, y, z) \cdot U_{n}
$$


with $E_{n}$ the precomputed map considering an unitary potential on the electrode $n$ and $U_{n}$ the potential applied on it. The dielectrophoretic force is then computed using Eq (6).

2) Superposition principle on the charge distribution: A second solution is to retrieve the electric field from the map of the charge accumulated on each electrode surface, leading to :

$$
E(x, y, z)=\frac{1}{2 \pi \varepsilon_{m}} \sum_{n=1}^{N} U_{n} \sum_{k=1}^{K} C_{n, k} \frac{\overrightarrow{P_{k} M}}{\left\|P_{k} M\right\|^{3}},
$$

where $C_{n, k}$ is the charge induced by the $n^{\text {th }}$ electrode on $P_{k}$, an elementary point. $M=(x, y, z)$ is the point of application.

\section{B. Analytical methods}

For particular geometry of electrodes, analytical formulations exist. For electrode arrays, Green's function [9] and Fourier series [10] have been recently studied.

1) Green's functions: The solution of the Laplace equation in a half-space, with Diriclet conditions, can be transformed, thanks to Green's theorem to:

$$
\phi(x, y, z)=\frac{z}{2 \pi} \int_{-\infty}^{\infty} \frac{h\left(x^{\prime}, y^{\prime}\right)}{\left[\left(x-x^{\prime}\right)^{2}+\left(y-y^{\prime}\right)^{2}+z^{2}\right]^{\frac{3}{2}}} d x^{\prime} d y^{\prime}
$$

with $\phi(x, y, z)$ the electric potential in the space and $h(x, y)$ the electric potential on the boundary. Gurtner et al explicit the electric potential on the boundary as a sum of polynomial approximations induced by each electrode $h(x, y)=$ $\sum_{n} \widetilde{h_{n}}(x, y) U_{n}$ [9]. The electric field can be derived using: $E(x, y, z)=-\nabla \phi(x, y, z)$.

2) Fourier series: Fourier series can be used to approximate the electric potential on the electrode array as a sum of exponential. The electric field in the whole space can be expressed as:

$$
E(x, y, z)=\sum_{n=1}^{N} \sum_{p} U_{n} a_{n, p} e_{p}(x, y, z),
$$

with $a_{n, p}$ the Fourier coefficient and $e_{p}(x, y, z)$ a combination of exponential. For the benchmark experiment, an analytical formulation of the $a_{n, p}$ coefficient is given in [10]. For more general cases, a semi-artificial neural network can be used to pre-compute numerical value of $a_{n, p}$ coefficient [17].

\section{Comparison of electric field computation techniques for closed loop control}

To be usable for closed loop control on an embedded platform, the approaches described above must provide accurate electric field computation while being resource efficient. In the following, the four previously presented methods are compared on the benchmark system presented in Figure 1. Since this system is invariant along the $\vec{x}$ direction, computation are reduced to $(O, \vec{y}, \vec{z})$ plan, significantly reducing the computation time.

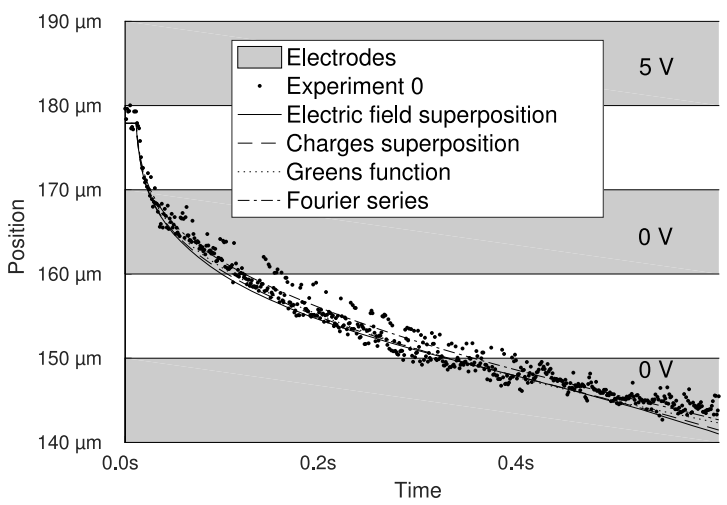

(a) Lateral position along $\vec{y}$ axis

Fig. 5: Position of the particle in the channel in response to a step voltage, experiment 0 . Different approaches to compute the electrical field are compared to experiment using dipolar approximation of the dielectrophoretic force and anisotropic drag force.

1) Precision: Using the same scenario as in Section III, the trajectory obtained with the four approaches to compute the electric field, enumerated previously, are compared to experimental trajectories. As discussed in the previous sections the anisotropic drag force and the dipolar model of the dielectrophoretic force are selected. For the semianalytical methods, maps of the electric field, its derivatives and charge distributions are pre-computed using a finite element software (Comsol). The Green's method uses third order polynomial approximation given in [9]. The Fourier method uses analytical formulation of the Fourier coefficients given in [10].

Figure 5 shows the prediction of the particle position with respect to time versus the experiments described in Section III before the particle hits the electrode array (bottom of the channel) at $t=0.6 \mathrm{~s}$. As dielectrophoresis is very sensitive to the voltage applied on each electrode, a quantitative study of the precision of each model requires comparison on a broad range of different electric fields in the whole space. For such exhaustive study, experimental data are hard to obtain. Thus, only qualitative conclusion is given here. The different model's trajectories laid on a bundle of $15 \mu \mathrm{m}$. This is less than twice the diameter of the particle. When performing closed loop control the inaccuracies in the model are handled by the feedback loop and can be considered as perturbations. Thus, the four models can be used to perform closed loop control. Computing resources, which are critical issues, are discussed in the next section.

2) Computing resources: The comparison of the computing resources required by these four approaches is been performed on three different arrays of respectively 8, 16 and 32 electrodes. In each case the electrode width is $10 \mu \mathrm{m}$. Thus, increasing the number of electrodes allows to compare the scale-up efficiency of each model. All models are run on the same desktop computer (Intel Core i5, $3.30 \mathrm{GHz}, 8 \mathrm{~GB}$ RAM, 64-bit, Linux 3.10, CentOS 7) from $\mathrm{C}$ code. $\mathrm{C}$ code is 
TABLE III: Time \& memory required to evaluate the dielectrophoretic force for different models on a 2D case

\begin{tabular}{|c|ll|ll|rr|cc|}
\hline Number of electrodes & \multicolumn{2}{|l|}{ Electric field superposition } & \multicolumn{2}{|l|}{ Charge distribution superposition } & \multicolumn{2}{|c|}{ Green's function } & Fourier series \\
\hline \hline $\mathbf{8}$ & $515 \mathrm{~ns}$, & $7.78 \mathrm{MB}$ & $55.0 \mu \mathrm{s}$, & $<2 \mathrm{MB}$ & $326 \mu \mathrm{s}$, & $<2 \mathrm{MB}$ & $21.9 \mu \mathrm{s}$, & $<2 \mathrm{MB}$ \\
\hline $\mathbf{1 6}$ & $605 \mathrm{~ns}$, & $30.0 \mathrm{MB}$ & $209 \mu \mathrm{s}$, & $<2 \mathrm{MB}$ & $616 \mu \mathrm{s}$, & $<2 \mathrm{MB}$ & $84.8 \mu \mathrm{s}$, & $<2 \mathrm{MB}$ \\
\hline $\mathbf{3 2}$ & $908 \mathrm{~ns}$, & $60.5 \mathrm{MB}$ & $812 \mu \mathrm{s}$, & $<2 \mathrm{MB}$ & $1.21 \mathrm{~ms}$, & $<2 \mathrm{MB}$ & $335 \mu \mathrm{s}$, & $<2 \mathrm{MB}$ \\
\hline
\end{tabular}

chosen as a reference for real-time embedded applications. The $\mathrm{C}$ code is available on request for each model. Table III shows the computing time and the memory required by the four models for the three different electrode arrays.

Based on these comparisons the electric field superposition method is the fastest, as it requires almost no computation. However, it requires a large amount of data loaded in memory. The charge superposition approach reduces the precomputed map to the electrodes surface, but it requires more computation leading to longer computation time. Green's function leads to complex formula using inverse tangent and square root, with a third order polynomial. For this reason this method is the slowest to compute. Fourier series are a good option when the number of electrode increases.

\section{Conclusions \& Perspectives}

This article aims at analyzing the performances of dynamic models usable for closed-loop control of dielectrophoretic systems. Dipolar model versus quadratic model of the dielectrophoretic force and isotropic versus anisotropic drag force model have been compared. We have shown that a model computing anisotropic drag force and dipolar dielectrophoretic force appears as the simplest model predicting trajectories close to the experiments. To compute the electric field, the method of superposition of the electrical field is the fastest to predict the electrical field. If memory consumption is an issue, Fourier series are a good option when the number of electrode increases.

The quadratic dependence of the dielectrophoretic force on the electric potential applied on each electrodes makes the inverse model hard to compute. Optimal closed-loop control will be investigated in future works.

\section{REFERENCES}

[1] G.-H. Lee, S.-H. Kim, K. Ahn, S.-H. Lee, and J. Y. Park, "Separation and sorting of cells in microsystems using physical principles," Journal of Micromechanics and Microengineering, vol. 26, no. 1, p. 013003, Jan. 2016.

[2] R. Pethig, "Review Article Dielectrophoresis: Status of the theory, technology, and applications," Biomicrofluidics, vol. 4, no. 2, June 2010.

[3] J. Zemánek, T. Michálek, and Z. Hurák, "Feedback control for noiseaided parallel micromanipulation of several particles using dielectrophoresis," Electrophoresis, vol. 36, pp. 1451-1458, July 2015.

[4] M. Kharboutly and M. Gauthier, "High speed closed loop control of a dielectrophoresis-based system," in 2013 IEEE International Conference on Robotics and Automation, May 2013, pp. 1446-1451.

[5] Z. Ni, S.-H. Ieng, C. Posch, S. Régnier, and R. Benosman, "Visual tracking using neuromorphic asynchronous event-based cameras," Neural Computation, vol. 27, no. 4, pp. 925-953, 2015.

[6] B. Brazey, J. Cottet, A. Bolopion, H. Van Lintel, P. Renaud, and M. Gauthier, "Impedance-based real-time position sensor for lab-ona-chip devices," Lab Chip, vol. 18, pp. 818-831, 2018.
[7] T. Michálek and J. Zemánek, "Dipole and multipole models of dielectrophoresis for a non-negligible particle size: Simulations and experiments," ELECTROPHORESIS, vol. 38, no. 11, pp. 1419-1426, June 2017.

[8] M. Kharboutly, M. Gauthier, and N. Chaillet, "Modeling the trajectory of a microparticle in a dielectrophoresis device," Journal of Applied Physics, vol. 106, no. 11, p. 114312, Dec. 2009.

[9] M. Gurtner, K. Hengster-Movric, and Z. Hurák, "Green's functionbased control-oriented modeling of electric field for dielectrophoresis," Journal of Applied Physics, vol. 122, no. 5, p. 054903, Aug. 2017.

[10] V. Gauthier, A. Bolopion, and M. Gauthier, "Analytical formulation of the electric field induced by electrode arrays: Towards automated dielectrophoretic cell sorting," Micromachines, vol. 8, no. 8, p. 253, Aug. 2017.

[11] A. Ambari, B. G. Manuel, and E. Guyon, "Effect of a plane wall on a sphere moving parallel to it," J. Phyique Lett., vol. 44, no. 4, pp. 143-146, Feb. 1983.

[12] A. Ambari, B. Gauthier-Manuel, and E. Guyon, "Wall effects on a sphere translating at constant velocity," Journal of Fluid Mechanics, vol. 149 , pp. 235-253, Dec. 1984.

[13] A. Castellanos, A. Ramos, A. González, N. G. Green, and H. Morgan, "Electrohydrodynamics and dielectrophoresis in microsystems: scaling laws," Journal of Physics D: Applied Physics, vol. 36, no. 20, pp. 2584-2597, Oct. 2003.

[14] X. Wang, X.-B. Wang, and P. R. Gascoyne, "General expressions for dielectrophoretic force and electrorotational torque derived using the Maxwell stress tensor method," Journal of electrostatics, vol. 39, no. 4, pp. 277-295, 1997.

[15] M. Washizu and T. B. Jones, "Multipolar dielectrophoretic force calculation," Journal of Electrostatics, vol. 33, no. 2, pp. 187-198, Sept. 1994.

[16] T. B. Jones, "Basic theory of dielectrophoresis and electrorotation," IEEE Engineering in Medicine and Biology Magazine, vol. 22, no. 6, pp. 33-42, Nov. 2003.

[17] H. Song and D. J. Bennett, "A semi-analytical approach using artificial neural network for dielectrophoresis generated by parallel electrodes," Journal of Electrostatics, vol. 68, no. 1, pp. 49-56, Feb. 2010. 